\title{
Small WebComputing Applied to Distributed Monte Carlo Calculations
}

\author{
P.A. Whitlock ${ }^{1}$, Dino Klein ${ }^{1}$, and Marvin Bishop ${ }^{2}$ \\ ${ }^{1}$ Department of Computer and Information Sciences, Brooklyn College \\ 2900 Bedford Avenue, Brooklyn, NY 11210-2889 \\ whitlock@its.brooklyn. cuny.edu \\ ${ }^{2}$ Department of Mathematics/Computer Science, Manhattan College \\ Riverdale, New York 10471 \\ marvin.bishop@manhattan.edu
}

\begin{abstract}
The software package, Small WebComputing (SWC), has been applied to a Monte Carlo simulation of a system of hard hyperspheres in a variety of dimensions. The SWC environment was chosen because once the framework is embedded in the application code, the user has the choice of running the distributed computations as a set of applets, as parallel threads on a symmetric multiprocessor or as independent processes distributed over a network. A description of the software and a discussion of its ongoing evolution is presented.
\end{abstract}

\section{Introduction}

The properties of hard hyperspherical systems in a variety of dimensions have been studied by us using Monte Carlo methods 1, 2, 3. In order to study systems with a minimum of several thousand hyperspheres a parallel or distributed computation was employed. The Small WebComputing (SWC) protocol developed by Ying and co-workers 4, 5, 6] was selected for the project because of several attractive features. Firstly, by simply incorporating several new classes into the Java simulation code, the actual distribution of the code is performed by the SWC software. The user is only responsible for the division of the calculation into tasks that can be run in parallel. Secondly, the distributed tasks can be run as Java applets and the sole requirement is that the host computer has the appropriate Java libraries, i.e. there is no need to upload the complete SWC software onto every host. Thirdly, the distributed tasks can also be run as threads or conventional distributed processes depending on the computers available.

An overview of the existing SWC software, a description of the Monte Carlo application, and a discussion of the ongoing improvements are given in this paper.

\section{The Original SWC Software}

The Small WebComputing framework was developed to provide master-worker MIMD parallel programming software for a user 5. It was meant to have an 
easy to use design that separated the programming interface from the underlying hardware. To achieve its goals, the SWC software was written in Java. The use of Java and Java applets provided inherent security through the "sandbox" security model [7, 8]. A set of extensible classes that sustain the framework for the user's computation were provided. In the original version of SWC, upper level communication, e.g. between the Master process and the server, used the Transmission Control Protocol (TCP) and lower level communication used the User Datagram Protocol (UDP). Eager scheduling [9, 10] was employed to guarantee load balancing and task completion.

The SWC framework envisions a Computation as composed of three levels: the Master, the Router, and the Worker components. A Computation that utilizes the original SWC framework must contain a Master, one or more Routers, and one or more Workers. The user programs the Master component so that it divides the computation into smaller, independent tasks, referred to as Work Units. The Workers are responsible for processing the Work Units (completing the task that the Work Unit represents) and returning Result Units to the Master. The Router component is provided by the software and its purpose is to act as a liaison between the Master and the Workers. All communication with the Workers is channeled through the Routers which protects the Master from being flooded by messages from Workers. Thus, the communication is tiered, with the Master only communicating with the Routers, the Routers communicating with both the Master and the Workers, and the Workers communicating only with the Routers (no inter-Worker transmission of data is allowed). Under eager scheduling Computations are guaranteed to finish. Any incomplete task, i.e. no Result Unit has yet been returned for the task, is kept on the task queues by the Routers and reassigned to idle Workers that request Work Units. The SWC software is completely thread based and therefore can easily take advantage of multiple CPU host computers.

In order to create a computation, the user must extend and implement the following abstract classes and interfaces:

SWCWorkUnit: a class implementing this interface will contain data regarding the task that needs to be done

SWCResultUnit: a class implementing this interface will contain results from the completed task

SWCMaster: the implementation of this abstract class must generate Work Units, collect Result Units, and output final computation results

SWCWorker: the implementation of this abstract class processes the Work Unit and creates a Result Unit which is returned to the Master.

It is important to note that the concrete class, SWCRouter, which functions as the Router in the system, does not need to be extended since it does not perform any computation specific operations. In the original version of SWC, the Router could not be shared among multiple computations because a Router was connected to one Master managing a single Computation. 


\section{Small WebComputing 2: An Improved Version of the Software}

The second version of the Small Web Computing (SWC2) framework[1] improved the usability of the software. Computation specificity was completely removed from the Master and Worker, and the framework hosted multiple, independent, concurrent Computations. The Router's functionality was still confined to transferring Work or Result Units among the Master and the Workers. This lack of specificity was achieved by incorporating a "pull model" of communication between each level of the framework. A Worker process requested (pulled) a Work Unit from a Router, while the Router requested Work Units from the Master. Computation completion was still guaranteed through eager scheduling.

SWC2 employed TCP for all communication between the components. The original SWC framework relied on UDP as the protocol for communicating with Workers. This choice, coupled with the inability to fragment Work or Result Units at the framework level, limited the size of Work or Result Units to 64 kilobytes. The decision to use UDP may have been due to the implementation of the TCP classes in the JDK version current when the SWC framework was originally written. In Table 3 of Ying, Arnow and Clark 4] which gives elapsed times for 100 iterations of transmissions of various data sizes, the TCP tests failed to transmit $4 \mathrm{~K}$ and $64 \mathrm{~K}$ data instances. Another difficulty with the use of UDP was the unreliable delivery of Work Units and Result Units. The SWC framework contained code to check periodically for the delivery of UDP packets. This code was the source of timing bottlenecks and bugs which hindered the early usages of the software. Since TCP guarantees delivery, the SWC2 framework does not need to test for delivery and perform retransmission of Work and Result Units. These changes simplified the software and lead to greater transparency of the SWC2 source code. There is no longer a restriction on the size of the data transmitted. Exhaustive testing has shown that there is no problem with using TCP for large data sets or frequently sent transmissions in SWC2.

\section{The Monte Carlo Application}

The behavior of multi-dimensional hyperspherical systems is governed by the Boltzmann distribution function, $f(\mathbf{R})$ :

$$
f(\mathbf{R})=\frac{\exp \left[-\sum \phi\left(r_{i j}\right) / k_{b} T\right]}{\int \exp \left[-\sum \phi\left(r_{i j}\right) / k_{b} T\right] d \mathbf{R}}
$$

where $\mathbf{R}$ is the d-dimensional vector, $\mathbf{r}_{i}=\left(x_{i 1}, x_{i 2}, \cdots, x_{i d}\right), i=1, \cdots, M$ of the $\mathrm{d}^{*} \mathrm{M}$ coordinates of the centers of mass of the $\mathrm{M}$ hyperspheres in the simulation box, $k_{b}$ is Boltzmann's constant and $\mathrm{T}$ is the absolute temperature of the system. The pair potential, $\phi\left(r_{i j}\right)$ represents the interaction between two hard hyperspheres; $r_{i j}=\left|\mathbf{r}_{\mathbf{i}}-\mathbf{r}_{\mathbf{j}}\right|$ and

$$
\phi_{h s}\left(r_{i j}\right)= \begin{cases}\infty, & \text { when } r_{i j}<\sigma \\ 0, & \text { when } r_{i j} \geq \sigma\end{cases}
$$


where $\sigma$ represents the contact distance between the two hyperspheres or the hypersphere diameter. The hyperspheres are initially placed in a simulation box on a lattice. The side lengths of this box are determined by the number of hyperspheres and the number density, $\rho$, the number of particles per unit volume.

The Metropolis 12 method was developed to assist in the Monte Carlo evaluation of integrals associated with complex physical systems. It guarantees the asymptotically correct sampling of an integrand, interpreted as a probability distribution function, by performing random walks in the many dimensional configuration space of the problem. These walks are generated by proposing a move from the current position of a hypersphere, $\mathbf{X}$, to a new position, $\mathbf{X}^{\prime}$. The new position is chosen from a probability distribution function, $H\left(\mathbf{X}^{\prime} \mid \mathbf{X}\right)$. In the present calculation, the new position is randomly chosen from a hyperbox surrounding the current position of the center of mass of the hypersphere. The proposed new position is then accepted or rejected based upon the probability $p\left(\mathbf{X}^{\prime} \mid \mathbf{X}\right)$ defined as:

$$
p\left(\mathbf{X}^{\prime} \mid \mathbf{X}\right)=\min \left(1, q\left(\mathbf{X}^{\prime} \mid \mathbf{X}\right)\right.
$$

where

$$
q\left(\mathbf{X}^{\prime} \mid \mathbf{X}\right)=\frac{H\left(\mathbf{X} \mid \mathbf{X}^{\prime}\right) f\left(\mathbf{X}^{\prime}\right)}{H\left(\mathbf{X}^{\prime} \mid \mathbf{X}\right) f(\mathbf{X})}
$$

If the new position is not accepted, the hypersphere remains at its current location. The acceptance ratio, the number of accepted moves divided by the number of total moves, is monitored. At higher densities, it is necessary to use much smaller trial moves in order to have a "reasonable" configuration change. Each pass of the random walk consists of one attempted move for each of the $\mathrm{M}$ hyperspheres. The move may or may not be accepted but is always counted in the averaging. As the random walk proceeds, a recursive relationship develops between the phenomenological distribution functions, $f_{n}(\mathbf{R})$, represented by each step of the random walk. As long as the system is ergodic and obeys detailed balance [12, 13, 14, $f_{n}(\mathbf{R}) \rightarrow f(\mathbf{R})$ is guaranteed to be true as $\mathrm{n}$, the number of passes, becomes large.

The Monte Carlo averaging procedure is performed to compute expectation values, $\langle A>$, of physical properties, $\mathrm{A}(\mathbf{R})$ :

$$
<A>=\frac{\int A(\mathbf{R}) f(\mathbf{R}) d \mathbf{R}}{\int f(\mathbf{R}) d \mathbf{R}} .
$$

In the Metropolis algorithm the successive positions of the hyperspheres are not independent; it takes many passes to converge from the initial state to an equilibrated one sampled from $f(\mathbf{R})$. Thus, some number of passes in the random walk must be discarded. Typically, on the order of $10^{3}-10^{4}$ passes are needed to reach the equilibrated state. Once the asymptotic distribution function is sampled, there is still serial correlation between each step in the random walk and this will affect the determination of the statistical error of the results. One method of dealing with these statistical correlations is to divide the random walks 
into blocks and use the block averages in the error analysis. Another approach is to perform totally independent sets of random walks and average the individual results together. The latter method is ideally suited for implementation with the SWC software.

Ying and co-workers 5 original vision was that the programmer would divide their computation into small tasks which would require an hour or two on any computer. This would not burden the volunteer host as it would likely be available for this amount of time. In the present case, for lower dimensional systems such as one or two dimensions, a random walk converges in one or two hours. The time for calculating a converged random walk increases dramatically in higher dimensions. A four dimensional system with 4096 hyperspheres typically took six hours and forty one minutes to complete ten parallel instances of a random walk consisting of thirteen thousand passes. However, an identically sized system in six dimensions, 4096 six dimensional hyperspheres, took thirty three hours to complete ten parallel instances of a random walk of eleven thousand passes at a similar number density. These large times for individual tasks require processors which are available for long periods of time and such tasks are probably not suited for running on widely scattered volunteer hosts.

While some of the calculations of the hard hypersphere systems have been deployed over a heterogeneous set of computers via the Internet, most of the production runs have been carried out on a network of Sun workstations. This mode of computation was selected because it was easier to harness a large group of networked workstations than to access a large number of volunteer computers. When volunteer hosts are requested to run the applet tasks, the version of the Java Virtual Machine running on each machine can be an issue. However, the applets did run successfully on PCs using Internet Explorer or Netscape running Windows, PCs using Mozilla running Linux and PCs using Netscape running Solaris. In the setting of separated colleagues collaborating on a calculation and thus able to negotiate the choice of browser and JVM, the applet version of the Worker is a feasible method of performing calculations.

When a computationally intensive code is written in Java, there is always a concern that the calculations will run substantially slower than if they were implemented in a different language. Extensive testing was done to compare the Java simulation code using the original SWC libraries against a serial $\mathrm{C}++$ code. The serial $\mathrm{C}++$ code and the parallel SWC code were run on the same processor and gave the exact same results. In a comparison of two, five dimensional runs involving 3125 hard hyperspheres with 3000 total passes, the serial code took 2 hours and 24 minutes, whereas the Java code with just one Worker took 2 hours. Similar timing results were obtained in all test cases, confirming our decision to use the SWC software.

\section{$5 \quad$ Evolution of SWC2 to Version 3}

After several years of experience with the software, major improvements are needed. In the SWC2 organization, no communication can occur directly between 
Workers. Each task has to be independent of the other tasks. To extend the hypersphere computation to dimensions greater than eight will require a major change in the way the the random walks are performed. The easiest alteration would be to divide each of the parallel random walks into tasks representing a thousand or so passes and to then serialize the random walk into sets of tasks. This would require the transmission of the intermediate positions of the hyperspheres in the random walk, between the Master and the Worker, to continue the walk. This can be accomplished with SWC2. An example of organizing a computation to allow for multiple exchanges of data between the Master and the Workers is given by the sample factorial computation in the SWC2 documentation[11. For very large numbers of particles, a commonly used alternative is to divide the domain space of the hard hypersphere system into subdomains containing sets of neighboring hyperspheres [15, 16. In the present Computations, the domain space is already partitioned into hypercells in order to efficiently detect hypersphere overlap. In a domain decomposition paradigm, the subdomains would represent distributed tasks and could be run on different Workers. If a hypersphere moved sufficiently far, it would need to be transferred to a different subdomain. Since the originating Worker would not know the location of the receiving Worker, a broadcast to all Workers might be needed. Alternatively, a barrier might be necessary to ensure that the Workers are approximately all at the same step in the random walk before a hypersphere is transferred. Workers would then need to check for a message at every move 17 . This is a well-known problem in parallel simulations 18, and can lead to great inefficiencies in the simulation [19].

The most fundamental change in the newest version, SWC3, is the communication system. It is being modified from a "pull model" to a fully asynchronous system. Messages can be sent between the Master and the Workshops, which manage workers. These messages can be control messages or other kinds of messages sent among Computations and Workshops.

The system allows, to limited extent, for communication to occur between a Computation and its Workshops, or among the Workers within a given Workshop. Restrictions on the type of communication are required in order to sustain the assumption that Work Units processed by different Workers, at different points in time, will produce results that are statistically identical.

These new communication capabilities are supported by the Router component, which exhibits enhanced functionality and responsibility. The Routers in the SWC3 system now communicate among themselves in order to locate Workers for inter-Worker messaging. Since reliability is not required for inter-router queries, the faster and more compact UDP protocol is used. Worker messages are still reliably sent using TCP connections.

Another benefit of the new communication system using control messages is the ability to terminate Workers on demand. In the previous version, SWC2, it was impossible to terminate specific Workers. If a Computation finished, but some of its replicated Work Units were still being processed, the user would have to wait needlessly. The user's alternative was to terminate the SWCWorker process, with the risk of negatively impacting another Computation. When a 
Computation terminates in SWC3, a notification message is sent to all Routers, which is subsequently transmitted to all Workshops. The notification causes Routers to dispose of any Work Units created by the now completed Computation, while Workshops will terminate any associated Workers.

Finally, a new interface is being developed in which Computations may periodically checkpoint, i.e. save a snapshot of their state. The purpose of this new functionality is to reduce the amount of lost work in the event that the Master terminates unexpectedly because if a Master is deleted, all the Computations are lost.

\section{Conclusion}

The Small WebComputing framework has been successfully used in the investigation of large hypersphere systems distributed on local area networks. To achieve the next stage of the investigation, dimensions higher than eight and tens of thousands of hyperspheres, requires changes in the organization of the computation code. How well this will work over the Internet with its inherent communication latency and a higher frequency of lost tasks needs careful evaluation.

\section{Acknowledgments}

We wish to thank the Brooklyn College Computing Center and the Manhattan College Computing Center for their support. One of us, D.K., was partially supported by PSC/CUNY Award \# 65358-0036.

\section{References}

1. Whitlock, P.A., Klein, D., and Bishop, M.: A parallel Monte Carlo Simulation of a 5-Dimensional hard sphere system using SWC. CUNY Ph.D. Program in Computer Science Technical Report, TR-200405, http://www.cs.gc.cuny.edu/tr/

2. Bishop, M., Whitlock, P.A. and Klein, D.: The Structure of Hyperspherical Fluids in Various Dimensions. J. Chem. Phys. 122 (2005) 074508

3. Bishop, M. and Whitlock, P.A.: The Equation of State of Hard Hyperspheres in Four and Five Dimensions. J. Chem. Phys. 123 (2005) 014507

4. Ying, K., Arnow, D. and D. Clark: Evaluating Communication Protocols for WebComputing. In Proceedings of the 1999 International Conference on Parallel and Distributed Processing Techniques and Applications, CSREA Press, Las Vegas, June (1999)

5. Arnow, D., Weiss, G., Ying, K. and Clark, D.: SWC:A Small Framework for WebComputing. In Proceedings of the International Conference on Parallel Computing, Delft, Netherlands, August (1999)

6. Ying, K.M.: WebComputing: Design and Performance. Ph.D. dissertation, Computer Science, City University of New York (2000)

7. http://java.sun.com/sfaq/verifier.html

8. McGraw, G. and Felten,E.W.: Securing Java: Getting Down to Business with Mobile Code, 2nd Edition. John Wiley \& Sons, New York, (1999) Chapter 2. 
9. Baratloo, A., Karaul, M., Kedem, Z.M. and Wijckoff, P.: Charlotte: Metacomputing on the Web. Future Generation Computer Systems. 15 (1999) 559

10. Neary, M.O. and Cappello, P.: Advanced Eager Scheduling for Java-Based Adaptively Parallel Computing. In Proc. of the 2002 Joint ACM - ISCOPE Conference on Java Grande (2002) 56

11. http://www.sci.brooklyn.cuny.edu/w̃whitlock/swc2docs/

12. Metropolis, N., Rosenbluth, A.W., Rosenbluth, M.N., Teller, A.H. and Teller, E.: Equations of state calculations by fast computing machines. J. Chem. Phys. 21 (1953) 1087

13. M.H. Kalos and P.A. Whitlock, Monte Carlo Methods, John Wiley and Sons, Inc., New York, 1986, pp. 73-86.

14. W.W. Wood, Monte Carlo studies of simple liquid models, in: H.N.V. Temperley, J.S. Rowlinson and G.S. Rushbrooke, (Eds.), The Physics of Simple Liquids, NorthHolland, Amsterdam, 1968, Chapter 5.

15. Barnes, J.E. and Hut, P.: A Hierarchical $\mathrm{O}(\mathrm{N} \log \mathrm{N})$ Force Calculation Algorithm. Nature 324 (1986) 446-449

16. Greengard, L. and Rokhlin, V.: A Fast Algorithm for Particle Simulations. J. Comp. Phys 73 (1987) 325-348

17. Wilkinson, B. and Allen, M.: Parallel Programming, Second Edition. Pearson Prentice Hall, Upper Saddle River, NJ (2005) Chapter 6

18. Jefferson, D.R.: Virtual Time, ACM Transactions on Programming Languages and Systems 7 (1985) 404-425

19. Jones, K. and Das, S.R.: Combining Optimism Limiting Schemes in Time Warp Based Parallel Simulations. In: D.J. Medeiros, E.F. Watson, J.S. Carson and M.S. Manivannan, eds., Proceedings of the 1998 Winter Simulation Conference 